Short Communication

\title{
Molecular data highlight hybridization in squirrel monkeys (Saimiri, Cebidae)
}

\author{
Jeferson Carneiro ${ }^{1}$, Luis Fernando da Silva Rodrigues-Filho ${ }^{1}$, Horacio Schneider ${ }^{1}$ and Iracilda Sampaio $^{1}$ \\ ${ }^{1}$ Universidade Federal do Pará, Campus Universitário de Bragança, Bragança, PA, Brazil.
}

\begin{abstract}
Hybridization has been reported increasingly frequently in recent years, fueling the debate on its role in the evolutionary history of species. Some studies have shown that hybridization is very common in captive New World primates, and hybrid offspring have phenotypes and physiological responses distinct from those of the "pure" parents, due to gene introgression. Here we used the TA15 Alu insertion to investigate hybridization in the genus Saimiri. Our results indicate the hybridization of Saimiri boliviensis peruviensis with S. sciureus macrodon, and S. b. boliviensis with $S$. ustus. Unexpectedly, some hybrids of both $S$. boliviensis peruviensis and $S$. b. boliviensis were homozygous for the absence of the insertion, which indicates that the hybrids were fertile.
\end{abstract}

Keywords: Saimiri, squirrel monkeys, Alu elements, hybridization.

Received: May 1, 2016; Accepted: August 11, 2016.

The Neotropical squirrel monkey genus Saimiri is one of the many platyrrhine taxa subject to controversy and uncertainty in terms of its species diversity and phylogenetic relationships (Alfaro et al., 2015). This genus is distributed primarily in the Amazon basin and Guianas (Figure 1), except Saimiri oerstedii, which is found in Central America, Costa Rica and Panama (Chiou et al., 2011). One of the most recent biogeographical studies of Saimiri (Alfaro et al., 2015) indicated that the species diversity of the genus is the product of a recent pan-Amazonian radiation. Based on the 14 clades identified in the analysis of the mitochondrial DNA (D-loop and cytb), these authors suggested a provisional taxonomy consisting of S. sciureus, S. oerstedii (S. o. oerstedii and S. o. citronellus), S. collinsi, S. ustus (A, B, and C lineages), S. boliviensis, S. cassiquiarensis (S. c. cassiquiarensis, S. c. albigena, S. c. macrodon A, S. c. macrodon B, and S. c. macrodon C), and S. vanzolinii. Analyzing mitochondrial DNA (CoxI and CoxII), Ruiz-García et al. (2015) proposed the following classification: $S$. oerstedii, with two subspecies $(S$. o. oerstedii and $S$. o. citrinellus), $S$. vanzolinii and $S$. sciureus, with two subspecies, S. s. boliviensis [with two lineages: 1 (boliviensis) and 2 (peruviensis)] and S. s. sciureus [with 12 lineages: 1 (sciureus), 2 (cassiquiarensis), 3 (ustus $\mathrm{I}=\mathrm{A}), 4$ (ustus $\mathrm{II}=$ B), 5 (ustus $\mathrm{III}=\mathrm{C}), 6($ macrodon $\mathrm{I}=\mathrm{D}), 7$ (macrodon $\mathrm{II}=$ E), 8 (macrodon III $=$ F), 9 (macrodon IV $=$ G), 10 (macrodon $\mathrm{V}=\mathrm{H}), 11$ (collinsi) and 12 (albigena)].

Saimiri populations occupy ample geographic areas (Figure 1), with many potential zones of contact that pro-

Send correspondence to Horacio Schneider. Campus Universitário de Bragança, Universidade Federal do Pará, Alameda Leandro Ribeiro 01, 68600000 Bragança, PA, Brazil. E-mail: horacio@ufpa.br. vide opportunities for hybridization between neighboring taxa (Hershkovitz, 1984). Thorington Jr (1985) reported cases of hybridization between $S$. ustus and S. sciureus on the east bank of the Tapajós River. Silva et al. (1992) investigated 49 specimens from a region in Peru occupied by both $S$. b. peruviensis and $S$. s. macrodon. By analyzing biochemical markers, these authors found clear evidence of admixture in approximately $45 \%$ of the individuals. Costello et al. (1993) also reported hybrids between $S$. ustus and $S$. sciureus from a region between the Madeira and Tapajós rivers.

Natural hybridization is the subject of a great deal of debate due to its potential importance as an evolutionary mechanism, especially for speciation, in addition to its relevance for taxonomy, conservation and species extinction (Mallet, 2005, 2007; Genovart, 2009;). Hybridization is known to have played a role in the evolutionary history of at least one quarter of plants and $10 \%$ of animal species (Rieseberg, 1997; Seehausen, 2004). Arnold and Meyer (2006) concluded that reticulate evolution is a common process in the evolutionary history of animals, with numerous examples of the formation of new taxa as a consequence of introgressive hybridization. In primates, this phenomenon has been reported in both captivity and the natural environment (Schreiber et al., 1998; Zinner et al., 2009; Matauschek et al., 2011). However, the exact role of hybridization in the evolutionary history of an organism is usually unclear, and reticulate evolution represents a potential pitfall for phylogenetic reconstructions. Arnold and Meyer (2006) suggested that the accuracy of some phylogenetic constructs of New World monkeys is probably weakened by hybridization events that occurred in the past. While it is difficult to detect hybridization events, 


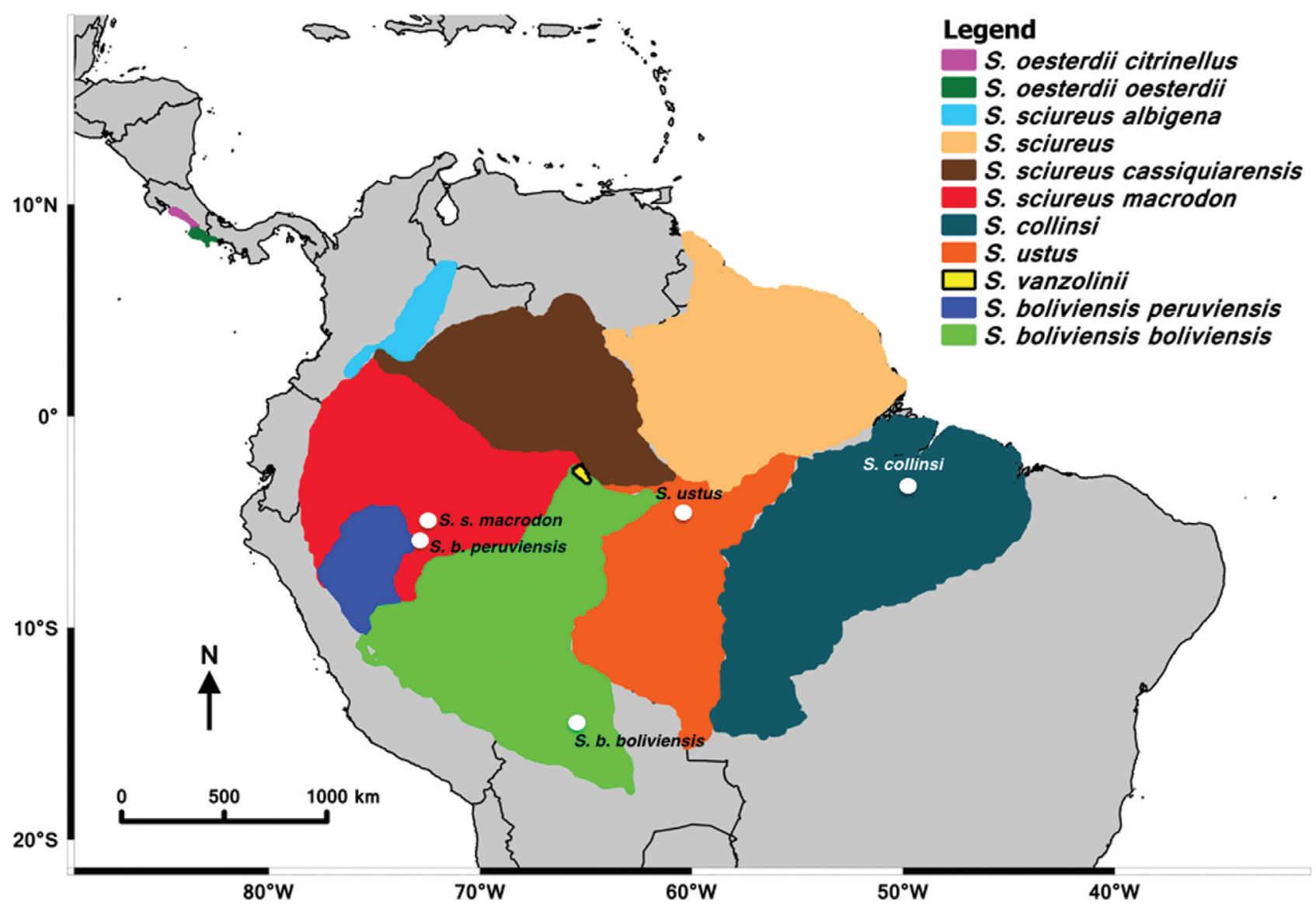

Figure 1 - Map showing the geographical distributions of Saimiri species and the original sites where each one of the five populations studied was found. The majority of S. b. boliviensis specimens where captured in Santa Cruz de La Sierra, Bolivia (region in green) and later transported to CAPRIM in Argentina. Those from S. b. peruviensis (region in blue) and S. sciureus macrodon species (region in red) where captured in the vicinity of Iquitos, Peru, and then transported to CRCP/IVITA in Iquitos. S. ustus (region in orange) and $S$. collinsi specimens (region in aquamarine blue) were captured and sampled in the forest.

Osterholz et al. (2008) described the integration of an Alu element in $S$. boliviensis, which is absent in S. sciureus.

In the human genome, Alu elements are the most abundant transposable features (Kriegs et al., 2007), and these elements are now known to comprise approximately $10 \%$ of the primate genome (Batzer and Deininger, 2002; Zhang et al., 2002). Once inserted into the genome of a species during its evolutionary history, Alu insertions will be present in all the descendants of that species. An Alu insertion is thus a single and irreversible event (Hamdi et al., 1999; Shedlock and Okada, 2000; Salem et al., 2003), and represents a marker free of homoplasies. The present study investigated the potential occurrence of hybridization in free-living populations of $S$. boliviensis, based on the presence or absence of AluTA15, as described by Osterholz et al. (2008).

We examined 107 samples of Saimiri: two $S$. sciureus macrodon, 16 S. collinsi, 17 S. ustus, 22 S. boliviensis peruviensis and 50 S. b. boliviensis (Table 1). All the individuals sampled were born in the wild, although in some cases, the blood samples were collected in captivity. The samples of $S$. collinsi were collected from animals captured during the rescue operation of the UHE Tucurui hydroelectric reservoir in Para, Brazil (La Rovere and Mendes, 2000), and those of S. ustus at UHE Samuel, in Rondonia (Fearnside, 2005). The samples of $S . b$. boliviensis, $S . b$. peruviensis and S. s. macrodon were obtained from two captive facilities, the "Centro de Reproducción y Conservación de Primates No Humanos" (CRCP/IVITA) in Iquitos, Peru, and the "Centro Argentinode Primates" (CAPRIM) in Corrientes, Argentina. The species were identified based on the morphological characteristics described by Hershkovitz (1984). S. b. boliviensis has a white zone around the eyes exhibiting sparse white hairs and a flattened arch over the eyes (roman arch) while in $S . s$ macrodon the arch formed above each eye is more evident and has been named as a "gothic arch".

While $S$. $b$. boliviensis and $S$. $b$. peruviensis have an arch that is less pronounced over the eyes (roman arch), $S$. b. peruviensis has a crown pattern on the head which is less eumelanized than that of $S$. b. boliviensis. The specimens held at CRCP/IVITA were classified as $S$. boliviensis peruviensis (roman arch) and those from the vicinity of Iquitos (Figure 1) as $S$. sciureus macrodon (gothic arch), 
Table 1 - Species, specimen code, locality, geographical coordinates and origin of the specimens analyzed in the present study.

\begin{tabular}{|c|c|c|c|c|c|}
\hline \multirow{2}{*}{$\begin{array}{l}\text { Taxa } \\
\text { Saimiri boliviensis boliviensis }\end{array}$} & \multirow{2}{*}{$\begin{array}{l}\text { Code } \\
\text { SBB } 2103\end{array}$} & \multirow{2}{*}{$\begin{array}{l}\text { Locality } \\
\text { Santa Cruz de La Sierra, Bolivia }\end{array}$} & \multicolumn{2}{|c|}{ Coordinates } & \multirow{2}{*}{$\begin{array}{l}\text { Origin } \\
\text { CAPRIM }\end{array}$} \\
\hline & & & $17^{\circ} 20^{\prime}$ & $64^{\circ} 03^{\prime}$ & \\
\hline Saimiri boliviensis boliviensis & SBB 2104 & Santa Cruz de La Sierra, Bolivia & $17^{\circ} 20^{\prime}$ & $64^{\circ} 03^{\prime}$ & CAPRIM \\
\hline Saimiri boliviensis boliviensis & SBB 2105 & Santa Cruz de La Sierra, Bolivia & $17^{\circ} 20^{\prime}$ & $64^{\circ} 03^{\prime}$ & CAPRIM \\
\hline Saimiri boliviensis boliviensis & SBB 2111 & Santa Cruz de La Sierra, Bolivia & $17^{\circ} 20^{\prime}$ & $64^{\circ} 03^{\prime}$ & CAPRIM \\
\hline Saimiri boliviensis boliviensis & SBB 2112 & Santa Cruz de La Sierra, Bolivia & $17^{\circ} 20^{\prime}$ & $64^{\circ} 03^{\prime}$ & CAPRIM \\
\hline Saimiri boliviensis boliviensis & SBB 2113 & Santa Cruz de La Sierra, Bolivia & $17^{\circ} 20^{\prime}$ & $64^{\circ} 03^{\prime}$ & CAPRIM \\
\hline Saimiri boliviensis boliviensis & SBB 2114 & Santa Cruz de La Sierra, Bolivia & $17^{\circ} 20^{\prime}$ & $64^{\circ} 03^{\prime}$ & CAPRIM \\
\hline Saimiri boliviensis boliviensis & SBB 2115 & Santa Cruz de La Sierra, Bolivia & $17^{\circ} 20^{\prime}$ & $64^{\circ} 03^{\prime}$ & CAPRIM \\
\hline Saimiri boliviensis boliviensis & SBB 2116 & Santa Cruz de La Sierra, Bolivia & $17^{\circ} 20^{\prime}$ & $64^{\circ} 03^{\prime}$ & CAPRIM \\
\hline Saimiri boliviensis boliviensis & SBB 2118 & Santa Cruz de La Sierra, Bolivia & $17^{\circ} 20^{\prime}$ & $64^{\circ} 03^{\prime}$ & CAPRIM \\
\hline Saimiri boliviensis boliviensis & SBB 2119 & Santa Cruz de La Sierra, Bolivia & $17^{\circ} 20^{\prime}$ & $64^{\circ} 03^{\prime}$ & CAPRIM \\
\hline Saimiri boliviensis boliviensis & SBB 2120 & Santa Cruz de La Sierra, Bolivia & $17^{\circ} 20^{\prime}$ & $64^{\circ} 03^{\prime}$ & CAPRIM \\
\hline Saimiri boliviensis boliviensis & SBB 2121 & Santa Cruz de La Sierra, Bolivia & $17^{\circ} 20^{\prime}$ & $64^{\circ} 03^{\prime}$ & CAPRIM \\
\hline Saimiri boliviensis boliviensis & SBB 2123 & Santa Cruz de La Sierra, Bolivia & $17^{\circ} 20^{\prime}$ & $64^{\circ} 03^{\prime}$ & CAPRIM \\
\hline Saimiri boliviensis boliviensis & SBB 2124 & Santa Cruz de La Sierra, Bolivia & $17^{\circ} 20^{\prime}$ & $64^{\circ} 03^{\prime}$ & CAPRIM \\
\hline Saimiri boliviensis boliviensis & SBB 2126 & Santa Cruz de La Sierra, Bolivia & $17^{\circ} 20^{\prime}$ & $64^{\circ} 03^{\prime}$ & CAPRIM \\
\hline Saimiri boliviensis boliviensis & SBB 2128 & Santa Cruz de La Sierra, Bolivia & $17^{\circ} 20^{\prime}$ & $64^{\circ} 03^{\prime}$ & CAPRIM \\
\hline Saimiri boliviensis boliviensis & SBB 2129 & Santa Cruz de La Sierra, Bolivia & $17^{\circ} 20^{\prime}$ & $64^{\circ} 03^{\prime}$ & CAPRIM \\
\hline Saimiri boliviensis boliviensis & SBB 2130 & Santa Cruz de La Sierra, Bolivia & $17^{\circ} 20^{\prime}$ & $64^{\circ} 03^{\prime}$ & CAPRIM \\
\hline Saimiri boliviensis boliviensis & SBB 2131 & Santa Cruz de La Sierra, Bolivia & $17^{\circ} 20^{\prime}$ & $64^{\circ} 03^{\prime}$ & CAPRIM \\
\hline Saimiri boliviensis boliviensis & SBB 2132 & Santa Cruz de La Sierra, Bolivia & $17^{\circ} 20^{\prime}$ & $64^{\circ} 03^{\prime}$ & CAPRIM \\
\hline Saimiri boliviensis boliviensis & SBB 2133 & Santa Cruz de La Sierra, Bolivia & $17^{\circ} 20^{\prime}$ & $64^{\circ} 03^{\prime}$ & CAPRIM \\
\hline Saimiri boliviensis boliviensis & SBB 2134 & Santa Cruz de La Sierra, Bolivia & $17^{\circ} 20^{\prime}$ & $64^{\circ} 03^{\prime}$ & CAPRIM \\
\hline Saimiri boliviensis boliviensis & SBB 2135 & Santa Cruz de La Sierra, Bolivia & $17^{\circ} 20^{\prime}$ & $64^{\circ} 03^{\prime}$ & CAPRIM \\
\hline Saimiri boliviensis boliviensis & SBB 2136 & Santa Cruz de La Sierra, Bolivia & $17^{\circ} 20^{\prime}$ & $64^{\circ} 03^{\prime}$ & CAPRIM \\
\hline Saimiri boliviensis boliviensis & SBB 2140 & Santa Cruz de La Sierra, Bolivia & $17^{\circ} 20^{\prime}$ & $64^{\circ} 03^{\prime}$ & CAPRIM \\
\hline Saimiri boliviensis boliviensis & SBB 2141 & Santa Cruz de La Sierra, Bolivia & $17^{\circ} 20^{\prime}$ & $64^{\circ} 03^{\prime}$ & CAPRIM \\
\hline Saimiri boliviensis boliviensis & SBB 2142 & Santa Cruz de La Sierra, Bolivia & $17^{\circ} 20^{\prime}$ & $64^{\circ} 03^{\prime}$ & CAPRIM \\
\hline Saimiri boliviensis boliviensis & SBB 2143 & Santa Cruz de La Sierra, Bolivia & $17^{\circ} 20^{\prime}$ & $64^{\circ} 03^{\prime}$ & CAPRIM \\
\hline Saimiri boliviensis boliviensis & SBB 2144 & Santa Cruz de La Sierra, Bolivia & $17^{\circ} 20^{\prime}$ & $64^{\circ} 03^{\prime}$ & CAPRIM \\
\hline Saimiri boliviensis boliviensis & SBB 2145 & Santa Cruz de La Sierra, Bolivia & $17^{\circ} 20^{\prime}$ & $64^{\circ} 03^{\prime}$ & CAPRIM \\
\hline Saimiri boliviensis boliviensis & SBB 2146 & Santa Cruz de La Sierra, Bolivia & $17^{\circ} 20^{\prime}$ & $64^{\circ} 03^{\prime}$ & CAPRIM \\
\hline Saimiri boliviensis boliviensis & SBB 2149 & Santa Cruz de La Sierra, Bolivia & $17^{\circ} 20^{\prime}$ & $64^{\circ} 03^{\prime}$ & CAPRIM \\
\hline Saimiri boliviensis boliviensis & SBB 2150 & Santa Cruz de La Sierra, Bolivia & $17^{\circ} 20^{\prime}$ & $64^{\circ} 03^{\prime}$ & CAPRIM \\
\hline Saimiri boliviensis boliviensis & SBB 2151 & Santa Cruz de La Sierra, Bolivia & $17^{\circ} 20^{\prime}$ & $64^{\circ} 03^{\prime}$ & CAPRIM \\
\hline Saimiri boliviensis boliviensis & SBB 2153 & Santa Cruz de La Sierra, Bolivia & $17^{\circ} 20^{\prime}$ & $64^{\circ} 03^{\prime}$ & CAPRIM \\
\hline Saimiri boliviensis boliviensis & SBB 2157 & Santa Cruz de La Sierra, Bolivia & $17^{\circ} 20^{\prime}$ & $64^{\circ} 03^{\prime}$ & CAPRIM \\
\hline Saimiri boliviensis boliviensis & SBB 2158 & Santa Cruz de La Sierra, Bolivia & $17^{\circ} 20^{\prime}$ & $64^{\circ} 03^{\prime}$ & CAPRIM \\
\hline Saimiri boliviensis boliviensis & SBB 2159 & Santa Cruz de La Sierra, Bolivia & $17^{\circ} 20^{\prime}$ & $64^{\circ} 03^{\prime}$ & CAPRIM \\
\hline Saimiri boliviensis boliviensis & SBB 2160 & Santa Cruz de La Sierra, Bolivia & $17^{\circ} 20^{\prime}$ & $64^{\circ} 03^{\prime}$ & CAPRIM \\
\hline Saimiri boliviensis boliviensis & SBB 2164 & Santa Cruz de La Sierra, Bolivia & $17^{\circ} 20^{\prime}$ & $64^{\circ} 03^{\prime}$ & CAPRIM \\
\hline Saimiri boliviensis boliviensis & SBB 2165 & Santa Cruz de La Sierra, Bolivia & $17^{\circ} 20^{\prime}$ & $64^{\circ} 03^{\prime}$ & CAPRIM \\
\hline Saimiri boliviensis boliviensis & SBB 2168 & Santa Cruz de La Sierra, Bolivia & $17^{\circ} 20^{\prime}$ & $64^{\circ} 03^{\prime}$ & CAPRIM \\
\hline Saimiri boliviensis boliviensis & SBB 2169 & Santa Cruz de La Sierra, Bolivia & $17^{\circ} 20^{\prime}$ & $64^{\circ} 03^{\prime}$ & CAPRIM \\
\hline Saimiri boliviensis boliviensis & SBB 2170 & Santa Cruz de La Sierra, Bolivia & $17^{\circ} 20^{\prime}$ & $64^{\circ} 03^{\prime}$ & CAPRIM \\
\hline Saimiri boliviensis boliviensis & SBB 2171 & Santa Cruz de La Sierra, Bolivia & $17^{\circ} 20^{\prime}$ & $64^{\circ} 03^{\prime}$ & CAPRIM \\
\hline
\end{tabular}




\begin{tabular}{|c|c|c|c|c|c|}
\hline \multirow{2}{*}{$\begin{array}{l}\text { Taxa } \\
\text { Saimiri boliviensis boliviensis }\end{array}$} & \multirow{2}{*}{$\begin{array}{l}\text { Code } \\
\text { SBB } 2174\end{array}$} & \multirow{2}{*}{$\begin{array}{l}\text { Locality } \\
\text { Santa Cruz de La Sierra, Bolivia }\end{array}$} & \multicolumn{2}{|c|}{ Coordinates } & \multirow{2}{*}{$\begin{array}{l}\text { Origin } \\
\text { CAPRIM }\end{array}$} \\
\hline & & & $17^{\circ} 20^{\prime}$ & $64^{\circ} 03^{\prime}$ & \\
\hline Saimiri boliviensis boliviensis & SBB 2176 & Santa Cruz de La Sierra, Bolivia & $17^{\circ} 20^{\prime}$ & $64^{\circ} 03^{\prime}$ & CAPRIM \\
\hline Saimiri boliviensis boliviensis & SBB $21 ? 5$ & Santa Cruz de La Sierra, Bolivia & $17^{\circ} 20^{\prime}$ & $64^{\circ} 03^{\prime}$ & CAPRIM \\
\hline Saimiri boliviensis boliviensis & SBB 21?? & Santa Cruz de La Sierra, Bolivia & $17^{\circ} 20^{\prime}$ & $64^{\circ} 03^{\prime}$ & CAPRIM \\
\hline Saimiri boliviensis peruviensis & SBP 1893 & East bank of the Marañón River, Peru & $06^{\circ} 41^{\prime}$ & $76^{\circ} 08^{\prime}$ & CRCP/IVITA \\
\hline Saimiri boliviensis peruviensis & SBP 1906 & East bank of the Marañón River, Peru & $06^{\circ} 41^{\prime}$ & $76^{\circ} 08^{\prime}$ & CRCP/IVITA \\
\hline Saimiri boliviensis peruviensis & SBP 1908 & East bank of the Marañón River, Peru & $06^{\circ} 41^{\prime}$ & $76^{\circ} 08^{\prime}$ & CRCP/IVITA \\
\hline Saimiri boliviensis peruviensis & SBP 1915 & East bank of the Marañón River, Peru & $06^{\circ} 41^{\prime}$ & $76^{\circ} 08^{\prime}$ & CRCP/IVITA \\
\hline Saimiri boliviensis peruviensis & SBP 1916 & East bank of the Marañón River, Peru & $06^{\circ} 41^{\prime}$ & $76^{\circ} 08^{\prime}$ & CRCP/IVITA \\
\hline Saimiri boliviensis peruviensis & SBP 1917 & East bank of the Marañón River, Peru & $06^{\circ} 41^{\prime}$ & $76^{\circ} 08^{\prime}$ & CRCP/IVITA \\
\hline Saimiri boliviensis peruviensis & SBP 1918 & East bank of the Marañón River, Peru & $06^{\circ} 41^{\prime}$ & $76^{\circ} 08^{\prime}$ & CRCP/IVITA \\
\hline Saimiri boliviensis peruviensis & SBP 1919 & East bank of the Marañón River, Peru & $06^{\circ} 41^{\prime}$ & $76^{\circ} 08^{\prime}$ & CRCP/IVITA \\
\hline Saimiri boliviensis peruviensis & SBP 1920 & East bank of the Marañón River, Peru & $06^{\circ} 41^{\prime}$ & $76^{\circ} 08^{\prime}$ & CRCP/IVITA \\
\hline Saimiri boliviensis peruviensis & SBP 1922 & East bank of the Marañón River, Peru & $06^{\circ} 41^{\prime}$ & $76^{\circ} 08^{\prime}$ & CRCP/IVITA \\
\hline Saimiri boliviensis peruviensis & SBP 1923 & East bank of the Marañón River, Peru & $06^{\circ} 41^{\prime}$ & $76^{\circ} 08^{\prime}$ & CRCP/IVITA \\
\hline Saimiri boliviensis peruviensis & SBP 1925 & East bank of the Marañón River, Peru & $06^{\circ} 41^{\prime}$ & $76^{\circ} 08^{\prime}$ & CRCP/IVITA \\
\hline Saimiri boliviensis peruviensis & SBP 1926 & East bank of the Marañón River, Peru & $06^{\circ} 41^{\prime}$ & $76^{\circ} 08^{\prime}$ & CRCP/IVITA \\
\hline Saimiri boliviensis peruviensis & SBP 1929 & East bank of the Maranon river, Peru & $06^{\circ} 41^{\prime}$ & $76^{\circ} 08^{\prime}$ & CRCP/IVITA \\
\hline Saimiri boliviensis peruviensis & SBP 1931 & East bank of the Maranon river, Peru & $06^{\circ} 41^{\prime}$ & $76^{\circ} 08^{\prime}$ & CRCP/IVITA \\
\hline Saimiri boliviensis peruviensis & SBP 1932 & East bank of the Marañón River, Peru & $06^{\circ} 41^{\prime}$ & $76^{\circ} 08^{\prime}$ & CRCP/IVITA \\
\hline Saimiri boliviensis peruviensis & SBP 1933 & East bank of the Marañón River, Peru & $06^{\circ} 41^{\prime}$ & $76^{\circ} 08^{\prime}$ & CRCP/IVITA \\
\hline Saimiri boliviensis peruviensis & SBP 1934 & East bank of the Marañón River, Peru & $06^{\circ} 41^{\prime}$ & $76^{\circ} 08^{\prime}$ & CRCP/IVITA \\
\hline Saimiri boliviensis peruviensis & SBP 1936 & East bank of the Maranon river, Peru & $06^{\circ} 41^{\prime}$ & $76^{\circ} 08^{\prime}$ & CRCP/IVITA \\
\hline Saimiri boliviensis peruviensis & SBP 1937 & East bank of the Maranon river, Peru & $06^{\circ} 41^{\prime}$ & $76^{\circ} 08^{\prime}$ & CRCP/IVITA \\
\hline Saimiri boliviensis peruviensis & SBP 1939 & East bank of the Marañón River, Peru & $06^{\circ} 41^{\prime}$ & $76^{\circ} 08^{\prime}$ & CRCP/IVITA \\
\hline Saimiri boliviensis peruviensis & SBP 1941 & East bank of the Marañón River, Peru & $06^{\circ} 41^{\prime}$ & $76^{\circ} 08^{\prime}$ & CRCP/IVITA \\
\hline Saimiri sciureus macrodon & SSM 1945 & $\begin{array}{l}\text { Ucayali River (Caserio Bagazan, Quebrada } \\
\text { Carahuayte), Peru }\end{array}$ & $07^{\circ} 52^{\prime}$ & $74^{\circ} 34^{\prime}$ & CRCP/IVITA \\
\hline Saimiri sciureus macrodon & SSM 1946 & $\begin{array}{l}\text { Ucayali River (Caserio Bagazan, Quebrada } \\
\text { Carahuayte), Peru }\end{array}$ & $07^{\circ} 52^{\prime}$ & $74^{\circ} 34^{\prime}$ & CRCP/IVITA \\
\hline Saimiri collinsi & $\mathrm{SC} 34$ & Left bank of the Tocantins River, Pará, Brazil & $03^{\circ} 52^{\prime}$ & $49^{\circ} 42^{\prime}$ & Free-living \\
\hline Saimiri collinsi & SC 36 & Left bank of the Tocantins River, Pará, Brazil & $03^{\circ} 52^{\prime}$ & $49^{\circ} 42^{\prime}$ & Free-living \\
\hline Saimiri collinsi & SC 410 & Left bank of the Tocantins River, Pará, Brazil & $03^{\circ} 52^{\prime}$ & $49^{\circ} 42^{\prime}$ & Free-living \\
\hline Saimiri collinsi & SC 473 & Left bank of the Tocantins River, Pará, Brazil & $03^{\circ} 52^{\prime}$ & $49^{\circ} 42^{\prime}$ & Free-living \\
\hline Saimiri collinsi & SC 525 & Left bank of the Tocantins River, Pará, Brazil & $03^{\circ} 52^{\prime}$ & $49^{\circ} 42^{\prime}$ & Free-living \\
\hline Saimiri collinsi & SC 626 & Left bank of the Tocantins River, Pará, Brazil & $03^{\circ} 52^{\prime}$ & $49^{\circ} 42^{\prime}$ & Free-living \\
\hline Saimiri collinsi & SC 627 & Left bank of the Tocantins River, Pará, Brazil & $03^{\circ} 52^{\prime}$ & $49^{\circ} 42^{\prime}$ & Free-living \\
\hline Saimiri collinsi & SC 686 & Left bank of the Tocantins River, Pará, Brazil & $03^{\circ} 52^{\prime}$ & $49^{\circ} 42^{\prime}$ & Free-living \\
\hline Saimiri collinsi & SC 749 & Left bank of the Tocantins River, Pará, Brazil & $03^{\circ} 52^{\prime}$ & $49^{\circ} 42^{\prime}$ & Free-living \\
\hline Saimiri collinsi & SC 847 & Left bank of the Tocantins River, Pará, Brazil & $03^{\circ} 52^{\prime}$ & $49^{\circ} 42^{\prime}$ & Free-living \\
\hline Saimiri collinsi & SC 863 & Left bank of the Tocantins River, Pará, Brazil & $03^{\circ} 52^{\prime}$ & $49^{\circ} 42^{\prime}$ & Free-living \\
\hline Saimiri collinsi & SC 865 & Left bank of the Tocantins River, Pará, Brazil & $03^{\circ} 52^{\prime}$ & $49^{\circ} 42^{\prime}$ & Free-living \\
\hline Saimiri collinsi & SC 873 & Left bank of the Tocantins River, Pará, Brazil & $03^{\circ} 52^{\prime}$ & $49^{\circ} 42^{\prime}$ & Free-living \\
\hline Saimiri collinsi & SC 1502 & Left bank of the Tocantins River, Pará, Brazil & $03^{\circ} 52^{\prime}$ & $49^{\circ} 42^{\prime}$ & Free-living \\
\hline Saimiri collinsi & SC 1549 & Left bank of the Tocantins River, Pará, Brazil & $03^{\circ} 52^{\prime}$ & $49^{\circ} 42^{\prime}$ & Free-living \\
\hline Saimiri collinsi & SC 1679 & Left bank of the Tocantins River, Pará, Brazil & $03^{\circ} 52^{\prime}$ & $49^{\circ} 42^{\prime}$ & Free-living \\
\hline Saimiri ustus & SU 2257 & Right bank of the Jamari River, Rondônia, Brazil & $08^{\circ} 56$ & $63^{\circ} 21^{\prime}$ & Free-living \\
\hline Saimiri ustus & SU 2305 & Right bank of the Jamari River, Rondônia, Brazil & $08^{\circ} 56$ & $63^{\circ} 21^{\prime}$ & Free-living \\
\hline
\end{tabular}




\begin{tabular}{llllll}
\hline Taxa & Code & Locality & Coordinates & Origin \\
\hline Saimiri ustus & SU 2354 & Right bank of the Jamari River, Rondônia, Brazil & $08^{\circ} 56$ & $63^{\circ} 21^{\prime}$ & Free-living \\
Saimiri ustus & SU 2450 & Right bank of the Jamari River, Rondônia, Brazil & $08^{\circ} 56$ & $63^{\circ} 21^{\prime}$ & Free-living \\
Saimiri ustus & SU 2454 & Right bank of the Jamari River, Rondônia, Brazil & $08^{\circ} 56$ & $63^{\circ} 21^{\prime}$ & Free-living \\
Saimiri ustus & SU 2577 & Right bank of the Jamari River, Rondônia, Brazil & $08^{\circ} 56$ & $63^{\circ} 21^{\prime}$ & Free-living \\
Saimiri ustus & SU 3193 & Right bank of the Jamari River, Rondônia, Brazil & $08^{\circ} 56$ & $63^{\circ} 21^{\prime}$ & Free-living \\
Saimiri ustus & SU 4030 & Right bank of the Jamari River, Rondônia, Brazil & $08^{\circ} 56$ & $63^{\circ} 21^{\prime}$ & Free-living \\
Saimiri ustus & SU 4031 & Left bank of the Jamari River, Rondônia, Brazil & $08^{\circ} 52$ & $63^{\circ} 15^{\prime}$ & Free-living \\
Saimiri ustus & SU 4032 & Left bank of the Jamari River, Rondônia, Brazil & $08^{\circ} 52$ & $63^{\circ} 15^{\prime}$ & Free-living \\
Saimiri ustus & SU 4033 & Left bank of the Jamari River, Rondônia, Brazil & $08^{\circ} 52$ & $63^{\circ} 15^{\prime}$ & Free-living \\
Saimiri ustus & SU 4041 & Left bank of the Jamari River, Rondônia, Brazil & $08^{\circ} 52$ & $63^{\circ} 15^{\prime}$ & Free-living \\
Saimiri ustus & SU 4257 & Left bank of the Jamari River, Rondônia, Brazil & $08^{\circ} 52$ & $63^{\circ} 15^{\prime}$ & Free-living \\
Saimiri ustus & SU 4441 & Left bank of the Jamari River, Rondônia, Brazil & $08^{\circ} 52$ & $63^{\circ} 15^{\prime}$ & Free-living \\
Saimiri ustus & SU 4508 & Left bank of the Jamari River, Rondônia, Brazil & $08^{\circ} 52$ & $63^{\circ} 15^{\prime}$ & Free-living \\
Saimiri ustus & SU 4550 & Left bank of the Jamari River, Rondônia, Brazil & $08^{\circ} 52$ & $63^{\circ} 15^{\prime}$ & Free-living \\
Saimiri ustus & SU 4577 & Left bank of the Jamari River, Rondônia, Brazil & $08^{\circ} 52$ & $63^{\circ} 15^{\prime}$ & Free-living \\
\hline
\end{tabular}

UHE Tucurui $=$ Tocantins River; UHE Samuel= Jamari River .

while the animals at CAPRIM, captured in Santa Cruz de La Sierra, Bolivia, were all S. boliviensis boliviensis (roman arch). Some of the animals at CAPRIM were born in captivity. Further details on the specimens and the geographical distribution of each population are presented in Table 1 and Figure 1. The material analyzed in the present study was part of the sample bank maintained by the Molecular Phylogenetics Laboratory at the Bragança campus of the Federal University of Para.

The total DNA was extracted using the Wizard Genomic kit (Promega, Madison, WI, USA) following the manufacturer's recommendations. The region of interest (AluTA15) was amplified using the primers and the protocol described by Osterholz et al. (2008). The initial denaturation step was 2 min at $94{ }^{\circ} \mathrm{C}$, followed by 40 cycles of denaturation $\left(1 \mathrm{~min}\right.$ at $\left.94^{\circ} \mathrm{C}\right)$, annealing $\left(1 \mathrm{~min}\right.$ at $\left.58{ }^{\circ} \mathrm{C}\right)$, and extension $\left(1 \mathrm{~min}\right.$ at $\left.72^{\circ} \mathrm{C}\right)$, with a final extension step of $5 \mathrm{~min}$ at $72{ }^{\circ} \mathrm{C}$. After amplification, the PCR products were separated electrophoretically in a $2 \%$ agarose gel at 60 $\mathrm{V}, 150 \mathrm{~mA}$ for $60 \mathrm{~min}$ together with a $1 \mathrm{~kb}$ plus DNA ladder (Invitrogen, Carsbad, CA, USA). All the fragments were stained with GelRed, as recommended by the manufacturer (Biotium, Hayward, CA, USA). Sequence reactions were conducted with a Big Dye v.3.1 kit (ABI BigDye ${ }^{\circledR}$ Terminator Mix; Applied Biosystems, Carlsbad, CA, USA), conducted in an ABI 3500xL sequencer (Applied Biosystems), to confirm that the region amplified by PCR was the fragment of interest (AluTA15). The sequences were aligned and edited manually in the BioEdit program (Hall, 1999).

The primers designed by Osterholz et al. (2008) amplify fragments of distinct sizes depending on the presence or absence of the Alu insertion (AluTA15). When the AluTA15 insertion is present, a fragment of approximately 750 base pairs (bps) is generated, but when it is absent, a fragment of only $450 \mathrm{bps}$ is generated. As the insertion is only present in S. boliviensis (Figure 2), in hybrids between this species and other Saimiri species, two fragments will be amplified, one with $750 \mathrm{bps}$ and another with $450 \mathrm{bps}$.

The AluTA15 insertion was not detected in any of the individuals identified as $S$. ustus $(\mathrm{n}=17)$ from Rondonia, $S$. collinsi $(\mathrm{n}=16)$ from Para or $S$. sciureus macrodon $(\mathrm{n}=2)$ from Peru. All 35 individuals presented only one band of approximately 450 bps (Table 2). By contrast, 50 specimens from Santa Cruz de La Sierra, Bolivia, identified as $S$. b. boliviensis, presented the insertion, of which $90 \%$ were homozygous $(+/+)$ and $10 \%$ (five individuals) were heterozygous (+/-) showing both bands (750 bps and $450 \mathrm{bps}$ ). This configuration was unexpected because Osterholz et al. (2008) proposed that the AluTA15 element was inserted into the lineage that originated the extant species $S$. boliviensis, which implies that all $S$. boliviensis should be homozygous for AluTA15 (+/+). Interestingly, all three possible combinations were found in the population previously identified as $S$. b. peruviensis from Peru (CRCP), with six individuals $(28 \%)$ being homozygous for the insertion $(+/+)$, eight (36\%) being homozygous for its absence $(-/-)$, and the other eight being heterozygous (+/-), showing both bands (750/450 bps) in the gel (Figure 2). Osterholz et al. (2008) also found three possible patterns of bands (+/+; $+/-;-/-)$ for specimens that were previously identified as $S$. b. peruviensis. So again, if the AluTA15 was inserted into the ancestral lineage of $S$. boliviensis, as proposed by Osterholz et al. (2008), it is unclear how specimens of this species could lack the insertion (-/-).

It is well known that $A l u$ elements are replicated in a copy-and-paste way in the primate genome, and once inserted into a genome, they cannot be excised. Given this, individuals phenotypically typical of Saimiri b. peruviensis, 


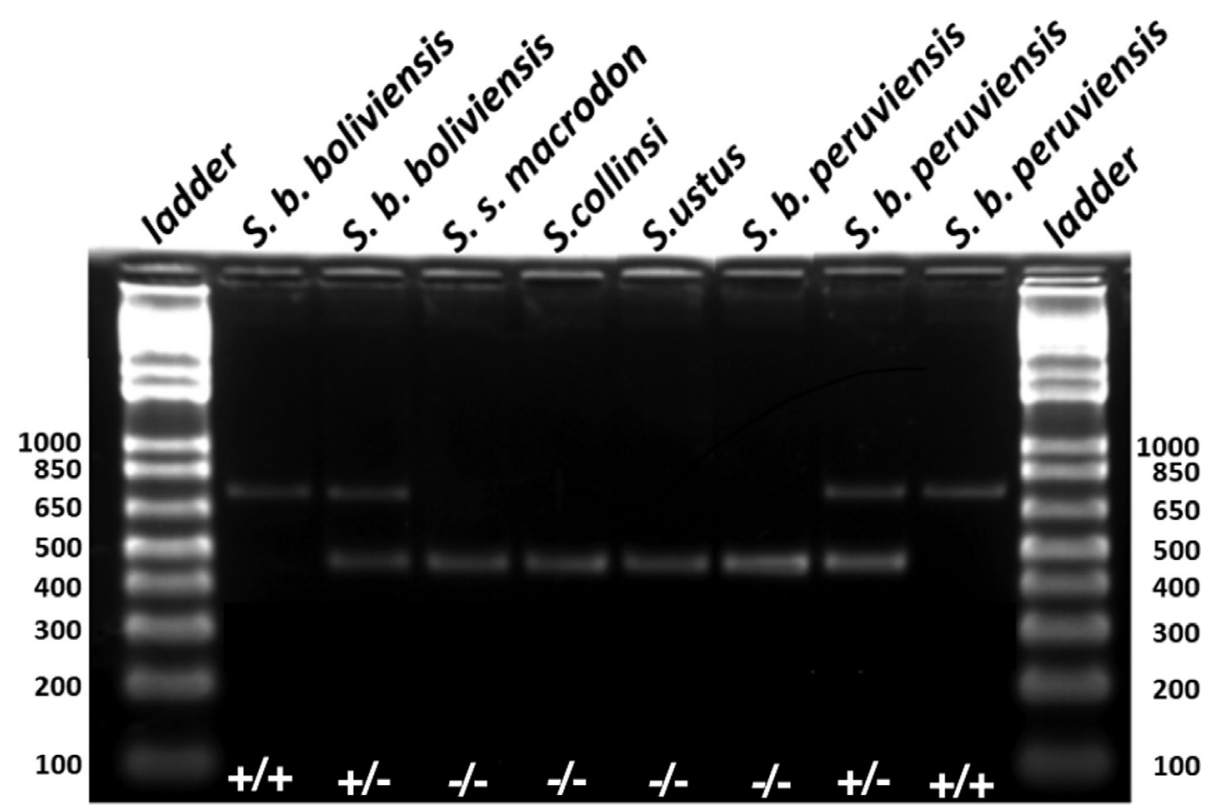

Figure 2 - Electrophoresis gel showing the distribution of the three Alu genotypes (+/+; +/-; and -/-) in the five subspecies sampled in the present study. A $1 \mathrm{~kb}$ ladder placed at both sides of the gel indicates the size in base pairs (bp) of the two amplified fragments.

Table 2 - Presence (+) or absence (-) of the Alu TA15 insertion in the Saimiri specimens analyzed in the present study.

\begin{tabular}{lcccc}
\hline & \multicolumn{3}{c}{ Number (\% of the total) of specimens: } \\
\cline { 2 - 4 } & Homozygous & Heterozygous & Homozygous & $+/+$ \\
Saimiri boliviensis peruviensis & $-/-$ & $-/+$ & $6(28 \%)$ & 22 \\
Saimiri boliviensis boliviensis & $8(36 \%)$ & $8(36 \%)$ & $45(90 \%)$ & 50 \\
Saimiri collinsi & 0 & $5(10 \%)$ & 0 & 16 \\
Saimiri sciureus macrodon & $16(100 \%)$ & 0 & 0 & 2 \\
Saimiri ustus & $2(100 \%)$ & 0 & 0 & 17 \\
Total: & $17(100 \%)$ & 13 & 51 & 107 \\
\hline
\end{tabular}

but heterozygous for the insertion $(+/-)$, must be the result of natural hybridization, which would presumably have involved the geographically closest taxon, S. sciureus macrodon. Furthermore, the absence of the insertion (-/-) in morphologically typical $S$. b. peruviensis can only be accounted for by the crossing of hybrid (+/-) Saimiri $b$. peruviensis or crosses between a hybrid and $S$. sciureus macrodon (-/-). These crosses would generate $25 \%$ or $50 \%$ of descendants without the insertion (-/-) and with dubious or intermediate morphological characteristics, which would represent conclusive evidence that hybridization between S. boliviensis and Saimiri sciureus macrodon produces fertile offspring. However, only $10 \%$ of the 50 Saimiri b. boliviensis specimens were heterozygous $(+/-)$, and probably originated from crosses with Saimiri ustus, due to the proximity of the geographical distribution of these species (Figure 2).

It is interesting to note that $S . b$. peruviensis and $S$. $s$. macrodon occur sympatrically in the region between the
Marañón and Tapiche rivers in the Peruvian Amazonia, whereas $S$. b. boliviensis is parapatric with $S$. s. macrodon and $S$. ustus, which are separated by the Juruá and PurusGuaporé Rivers, respectively (Hershkovitz, 1984). However, these rivers do not constitute an effective geographic barrier to gene flow in lizards (Souza et al., 2013), primates, and other organisms (Gascon et al., 2000), which implies that there may be gene flow between the present-day ranges of the three Saimiri species, resulting in hybridization between Saimiri boliviensis and Saimiri sciureus or S. ustus, as suggested by previous authors (Hershkovitz, 1984; Thorington Jr, 1985; Silva et al., 1992, 1993; Osterholz et al., 2008) based on morphological data.

Using chromosomal data, Jones and Ma (1975) were able to distinguish between $S$. b. peruviensis and $S$. $s$. macrodon from the vicinity of Iquitos (Peru) and Leticia (Colombia), respectively. Both species revealed a diploid number of $2 \mathrm{n}=42$, with $10 \mathrm{meta} /$ submetacentric, 22 acrocentric and 10 telocentric chromosomes in $S . b$. 
peruviensis, and $10 \mathrm{meta} / \mathrm{submetacentric,} 20$ acrocrentic, and 12 telocentric chromosomes in S. s. macrodon. A hybrid produced in the laboratory between a male from Iquitos and a female from Leticia showed 10 meta/submetacentric, 11 acrocentic and 11 telocentric chromosomes. Lau and Arrighi (1976) using chromosomal banding analyses detected two nonhomologous pericentric inversions in the telocentric group of chromosomes of a squirrel monkey, Saimiri sciureus, suggesting that this individual was an intersubspecific hybrid whose parents originated from different geographical locations. Recently, Ruiz-García et al. (2015) also found evidence of hybridization between Saimiri species based on mitochondrial markers (Cox1 and Cytb), emphasizing the importance of this process in the species-level diversification of this genus. In fact, these authors concluded that this genus comprises only three species, S. oerstedi, S. sciureus, and Saimiri vanzolinii, which diversified during the Pleistocene. This is consistent with the estimate of Alfaro et al. (2015), who concluded that $S$. boliviensis diverged from the other Saimiri species less than 1.5 Ma.

Hybridization may be a catalyst not only for speciation but also for major evolutionary innovations (Mallet (2007). Hybridization between Saimiri species appears to be common, and as Mallet (2007) concludes at page 182: "most speciation involves natural selection; natural selection requires genetic variation; genetic variation is enhanced by hybridization; and hybridization and introgression between species is a regular occurrence, especially in rapidly radiating groups". On the basis of the evidence presented here, this appears to have been the case in Saimiri.

\section{Acknowledgments}

This study was part of the MSc thesis of JC, which was supported by the Brazilian National Council for Scientific and Technological Development (CNPq). This research was also supported by the collaborative program,Dimensions US-Biota-São Paulo: Assembly and evolution of the Amazon biota and its environment: an integrated approach, supported by the US National Science Foundation (NSF), National Aeronautics and Space Administration (NASA), and the São Paulo State Research Foundation (FAPESP). Funds for this research were also provided by CNPq (grants 306233/2009-6 to IS, and 473341/2010-7, 305645/2009-9) and CAPES Program No. 3296/2013-PROAM to HS

\section{References}

Alfaro JWL, Boubli JP, Paim FP, Ribas CC, Da Silva MNF, Messias MR, Röhe F, Mercês MP, Júnior JSS and Silva CR (2015) Biogeography of squirrel monkeys (genus Saimiri): South-central Amazon origin and rapid pan-Amazonian diversification of a lowland primate. Mol Phylogenet Evol 82:436-454.
Arnold ML and Meyer A (2006) Natural hybridization in primates: One evolutionary mechanism. Zoology 109:261-276.

Batzer MA and Deininger PL (2002) Alu repeats and human genomic diversity. Nat Rev Genet 3:370-379.

Chiou KL, Pozzi L, Alfaro JWL and Di Fiori A (2011) Pleistocene diversification of living squirrel monkeys (Saimiri spp.) inferred from complete mitochondrial genome sequences. Mol Phylogenet Evol 59:736-745.

Costello RK, Dickinson C, Rosenberger AL, Boinski S and Szalay FS (1993) Squirrel monkey (genus Saimiri) taxonomy. In: Kimble WH and Martin LB (eds) Species, Species Concepts and Primate Evolution. Springer, Boston, pp 177-210.

Fearnside MP (2005) Brazil's Samuel Dam: Essons for hydroelectric development policy and the environment in Amazonia. Environ Manag 35:1-19.

Gascon C, Malcolm JR, Patton JL, Da Silva MN, Bogart JP, Lougheed SC, Peres CA, Neckel S and Boag PT (2000) Riverine barriers and the geographic distribution of Amazonian species. Proc Natl Acad Sci U.S.A. 97:13672-13677.

Genovart M (2009) Natural hybridization and conservation. Biodiv Conserv 18:1435-1439.

Hall TA (1999) BioEdit: A user-friendly biological sequence alignment editor and analysis program for Windows 95/98/NT. Nucleic Acids Symp Ser 41:95-98.

Hamdi H, Nishio H, Zielinski R and Dugaiczyk A (1999) Origin and phylogenetic distribution of Alu DNA repeats: irreversible events in the evolution of primates. J Mol Biol 289:861-871.

Hershkovitz P (1984) Taxonomy of squirrel monkeys genus Saimiri (Cebidae, Platyrrhini): A preliminary report with description of a hitherto unnamed form. Am J Primatol 6:257312.

Kriegs JO, Churakov G, Jurka J, Brosius J and Schmitz J (2007) Evolutionary history of 7SL RNA-derived SINEs in Supraprimates. Trends Genet 23:158-161.

Jones TC and Ma NSF (1975) Cytogenetics of the squirrel monkey (Saimiri sciureus). In: Goodwin WJ and Augustine J (eds) Primate Research. Springer, Boston, pp 13-21.

La Rovere E and Mendes F (2000) Tucuruí Hydropower Complex, Brazil. A WCD Case Study Prepared as an Input to the World Commission on Dams. Cape Town World Commission on Dams Secretariat, Cape Town, 195 p.

Lau Y-F and Arrighi FE (1976) Studies of the squirrel monkey, Saimiri sciureus, genome 1. Cytological characterization of chromosomal heterozygosity. Cytogenet Cell Genet 17:5160.

Mallet J (2005) Hybridization as an invasion of the genome. Trends Ecology Evol 20:229-237.

Mallet J (2007) Hybrid speciation. Nature 446:279-283.

Matauschek C, Roos C and Heymann EW (2011) Mitochondrial phylogeny of tamarins (Saguinus, Hoffmannsegg 1807) with taxonomic and biogeographic implications for the $S$. nigricollis species groupAm J Phys Anthropol 144:564-574.

Osterholz M, Vermeer J, Walter L and Roos C (2008) A PCRbased marker to simply identify Saimiri sciureus and $S$. boliviensis boliviensis. Am J Primatol 70:1177-1180.

Rieseberg LH (1997) Hybrid origins of plant species. Annu Rev Ecol Syst 28:359-389.

Ruiz-García M, Luengas-Villamil K, Leguizamon N, De Thoisy B and Gálvez H (2015) Molecular phylogenetics and phylo- 
geography of all the Saimiri taxa (Cebidae, Primates) inferred from $\mathrm{mt} \mathrm{COI}$ and $\mathrm{COII}$ gene sequences. Primates 56:145-161.

Salem A-H, Ray DA, Xing J, Callinan PA, Myers JS, Hedges DJ, Garber RK, Witherspoon DJ, Jorde LB and Batzer MA (2003) Alu elements and hominid phylogenetics. Proc Natl Acad Sci U S A 100:12787-12791.

Schreiber A, Wang M and Kaumanns W (1998) Captive breeding of squirrel monkeys, Saimiri sciureus and Saimiri boliviensis: the problem of hybrid groups. Zoo Biol 17:95109.

Seehausen O (2004) Hybridization and adaptive radiation. Trends Ecology Evol 19:198-207.

Shedlock A and Okada N (2000) SINE insertions: Powerful tools for molecular systematic. Bioessays 22:148-160.

Silva BTF, Sampaio MIC, Schneider H, Schneider MPC, Montoya E, Encarnacion F, Callegari Jacques SM and Salzano FM (1993) Protein electrophoretic variability in Saimiri and the question of its species status. Am J Primatol 29:183-193.

Silva BTF, Sampaio MIC, Schneider H, Schneider MPC, Montoya E, Encarnacion F and Salzano FM (1992) Natural hy- bridization between Saimiri taxa in the Peruvian Amazonia. Primates 33:107-113.

Souza SM, Rodrigues MT and Cohn-Haft M (2013) Are Amazonia rivers biogeographic barriers for lizards? A study on the geographic variation of the spectacled lizard Leposoma osvaldoi Ávila-Pires (Squamata, Gymnophthalmidae). J Herpetol 47:511-519.

Thorington Jr RW (1985) The taxonomy and distribution of squirrel monkeys (Saimiri). In: Rosenblum LA and Coe CL (eds) Handbook of Squirrel Monkey Research. Springer, New York, pp 1-33.

Zhang Z, Harrison P and Gerstein M (2002) Identification and analysis of over 2000 ribosomal protein pseudogenes in the human genome. Genome Res 12:1466-1482.

Zinner D, Groeneveld LF, Keller C and Roos C (2009) Mitochondrial phylogeography of baboons (Papio spp.) - Indication for introgressive hybridization? BMC Evol Biol 9:e83.

Associate Editor: Francisco Mauro Salzano

License information: This is an open-access article distributed under the terms of the Creative Commons Attribution License (type CC-BY), which permits unrestricted use, distribution and reproduction in any medium, provided the original article is properly cited. 